\title{
DIRETRIZES DE CRIAÇÃO DE TEMPLATES PARA MODELAGEM EM BIM COM BASE NO SINAPI: ESTUDO DOS MODELOS DE CUSTO
}

\author{
Isabela Andrade ${ }^{(1)}$, Aline Albuquerque ${ }^{(1)}$, Daniele do Nascimento ${ }^{(1)}$, Rodrigo \\ Fernandes $^{(1)}$
}

(1) Caixa Econômica Federal, Brasília

\begin{abstract}
Resumo
Este artigo relata modelagens em Building Information Modeling (BIM) desenvolvidas pela Caixa Econômica Federal (CAIXA) com foco na orçamentação de obras públicas. Essas atividades surgiram como resposta a questionamentos sobre integração entre BIM e o SINAPI. O objetivo do artigo é apresentar um método alternativo àquele que privilegia o detalhamento geométrico do modelo em detrimento da gestão da informação. A abordagem possibilita quantificação conforme referências do SINAPI, caracterizado por diversidade de parâmetros. $\mathrm{O}$ artigo descreve nomenclatura simplificada, que permite escolha de parâmetros em planilha, reduzindo a necessidade de detalhamento do modelo. Essas questões são trabalhadas empiricamente em estudo de caso, onde a aplicação da metodologia de parâmetros de texto mostrou-se útil para indicação de fatores de produtividade, de especificação de materiais e de geometria das peças. Neste estudo, apresenta-se metodologia de nomenclatura proveniente do SINAPI aliada ao emprego do BIM, em que se objetiva garantir agilidade na orçamentação. $\mathrm{O}$ estudo mostrou que a metodologia aplicada permite maior produtividade na elaboração de modelos, equilibrando esforço de modelagem e quantificação via ferramenta gerenciadora de informações. Isso permite construir um modelo consistente e suficiente, com poucos elementos e com Nível de Desenvolvimento menos complexo.
\end{abstract}

\section{Contextualização SINAPI}

O Sistema Nacional de Pesquisas de Custos e Índices da Construção Civil (SINAPI) é um sistema de referência de preços de insumos e custos de composições de serviços mantido pelas equipes da Caixa Econômica Federal (CAIXA) e do Instituto Brasileiro de Geografia e Estatística (IBGE). Foi criado em 1969 no âmbito da Política Habitacional promovida pelo Banco Nacional de Habitação. Com a extinção desse Banco, em 1986, o SINAPI passou a ficar sob gestão da CAIXA. A partir de 2003, devido a uma determinação legal do Governo Federal, o Sistema passou a ser de uso obrigatório para todas as obras executadas com recursos do 
Orçamento Geral da União (OGU). Desde então, manteve-se a obrigatoriedade do Sistema como balizador de custos para licitações e contratos de obras e serviços de engenharia que se utilizam de recursos do orçamento da União [1] e, recentemente, para as licitações de obras e serviços de engenharia realizados mediante contratos celebrados por empresas públicas e sociedades de economia mista [2]. O acesso ao SINAPI é público e gratuito, o que contribuiu para se tornar o principal sistema de referência de custos do setor da construção civil brasileiro. Suas referências de preços e custos são constantemente ampliadas e aperfeiçoadas pelas equipes das instituições públicas responsáveis por sua manutenção.

Enquanto ao IBGE cabem as atividades mensais de coleta, apuração e cálculo dos preços dos insumos - nas 27 Unidades da Federação - à CAIXA cabem as atividades técnicas de engenharia como, por exemplo, o desenvolvimento das composições unitárias de custo de serviços, a definição das especificações técnicas dos insumos e a elaboração dos Cadernos Técnicos de Composições.

Um marco na gestão técnica do Sistema, foi o processo de aferição das composições do SINAPI, iniciado em 2013. Esse processo vem atualizando as referências técnicas, além de tornar o conteúdo do Sistema mais transparente.

No contexto desses avanços técnicos, a CAIXA deu início em 2015 à publicação das Demonstrações de Uso das Referências SINAPI (conceito explicado no item 3) [3] com o objetivo de apresentar aos usuários do SINAPI exemplos do emprego das composições aferidas em projetos hipotéticos (em geral, tipologias de empreendimentos recorrentemente apresentadas à CAIXA para fins de análise nos processos de concessão de financiamento e de repasse de recursos públicos).

Para desenvolver esses modelos de exemplificação - do uso das composições do SINAPI - a equipe responsável tem recorrido à utilização de softwares BIM para a extração dos quantitativos dos serviços com maior confiabilidade e segurança. O grande desafio dessa tarefa tem sido desenvolver um método ágil de modelagem, que tire partido dos benefícios das ferramentas BIM e que considere a diversidade de parâmetros presentes nas referências de custo do SINAPI. É sobre esse desafio e as formas encontradas pela CAIXA para enfrentá-lo que trata o presente trabalho.

\section{Cenário Nacional do BIM e o SINAPI}

Em 2009, foi criada a Comissão de Estudos Especial de Modelagem de Informação da Construção (ABNT/CEE-134) com o objetivo de desenvolver normas técnicas relacionadas ao BIM, por meio de "tradução da norma ISO 12006-2; desenvolvimento de um sistema de classificação para a Construção e; desenvolvimento de diretrizes para criação de componentes BIM." [4]. Dentre outras iniciativas, destacam-se a instituição da Estratégia Nacional de Disseminação do BIM (Estratégia BIM BR) e a criação do Comitê Gestor da Estratégia do BIM para fomentar o uso e a difusão do BIM na construção civil brasileira [5] em 2018.

O processo de Demonstração de Uso das Referências SINAPI abordado no presente artigo é uma iniciativa da CAIXA para incorporar o BIM em seus processos, e se insere no contexto da coletânea de normas técnicas ABNT NBR 15965. O objetivo dessa norma, de acordo com [6], é oferecer termos e conceitos padronizados refletindo as práticas e sistemas construtivos utilizados no Brasil, viabilizando a chamada tropicalização de referências estrangeiras, essencialmente as normas ISO. A partir dos padrões estabelecidos pela norma (códigos e 
tabelas), seria possível combiná-los entre si de forma a representar todo e qualquer elemento da construção civil dentro das características brasileiras por meio de informação legível por computadores.

As normativas brasileiras atuais para projeto, orçamento, licitação, auditoria e medição de obras são baseadas em sistemas construtivos tradicionais, que contam com elevado emprego de mãode-obra, baixa aplicação de tecnologia, alta variação de formas de preparo de materiais. Neste contexto, é necessário avaliar diversos parâmetros, que geram um LOI (Level of Information) bastante elevado.

Diante desse cenário, a iniciativa da Demonstração de Uso das Referências SINAPI - pela CAIXA - ganha destaque e relevância considerando o seu potencial de gerar desdobramentos quanto à disseminação do BIM na CAIXA e no país, bem como de facilitar e popularizar a adoção do SINAPI em modelos BIM.

\section{As Demonstrações de Uso de Referências SINAPI: considerações sobre o conceito}

Neste trabalho, entende-se por Demonstração de Uso das Referências SINAPI, os modelos de informação da construção desenvolvidos por equipe técnica da CAIXA - em softwares BIM com o intuito estrito de exemplificar o emprego de composições aferidas do SINAPI em projetos habitacionais, de equipamentos públicos ou comunitários. Esses projetos são gentilmente cedidos à CAIXA, pelos autores ou órgãos públicos, unicamente para esse fim.

Após a captação desses projetos, a equipe da CAIXA inicia o processo de modelagem digital utilizando-se de software de modelagem BIM autoral. Inicialmente, a modelagem tem se restringido aos elementos de arquitetura e estrutura, devido ao fato de que a metodologia se encontra em desenvolvimento e, também, às características das composições dos serviços de instalações prediais do SINAPI. Além disso, para fins de modelagem de custos, não são considerados alguns itens dos projetos, conforme descrito no documento de Metodologia de Demonstração de Uso das Referências SINAPI [7].

Concluída a etapa de modelagem, passa-se à extração dos quantitativos. Cabe ressaltar, conforme previsto em [7], que todos os materiais e serviços incluídos no modelo de custos (e que correspondem a itens e subitens da planilha orçamentária) são provenientes dos bancos de insumos e composições de serviços do SINAPI sendo adotadas, preferencialmente, as composições já aferidas e detalhadas nos Cadernos Técnicos de cada grupo de serviços.

\section{Evolução do método de modelagem}

\subsection{Estrutura de codificação única do SINAPI}

O SINAPI é composto de diversos itens para a formação de custo. Cada item possui um código completo, e outro respectivo código numérico. A Figura 1 exemplifica o processo de geração deste código, sendo que: (i) a parte "01.PARE.ALVE" do código representa alvenaria de vedação de blocos cerâmicos furados na horizontal de 9x19,x19cm (espessura $9 \mathrm{~cm}$ ) de paredes com área líquida maior ou igual a $6 \mathrm{~m} 2$ com vãos; (ii) e a parte " 01 " e " 02 " correspondem a argamassa de assentamento com preparo de betoneira e manual, respectivamente. 


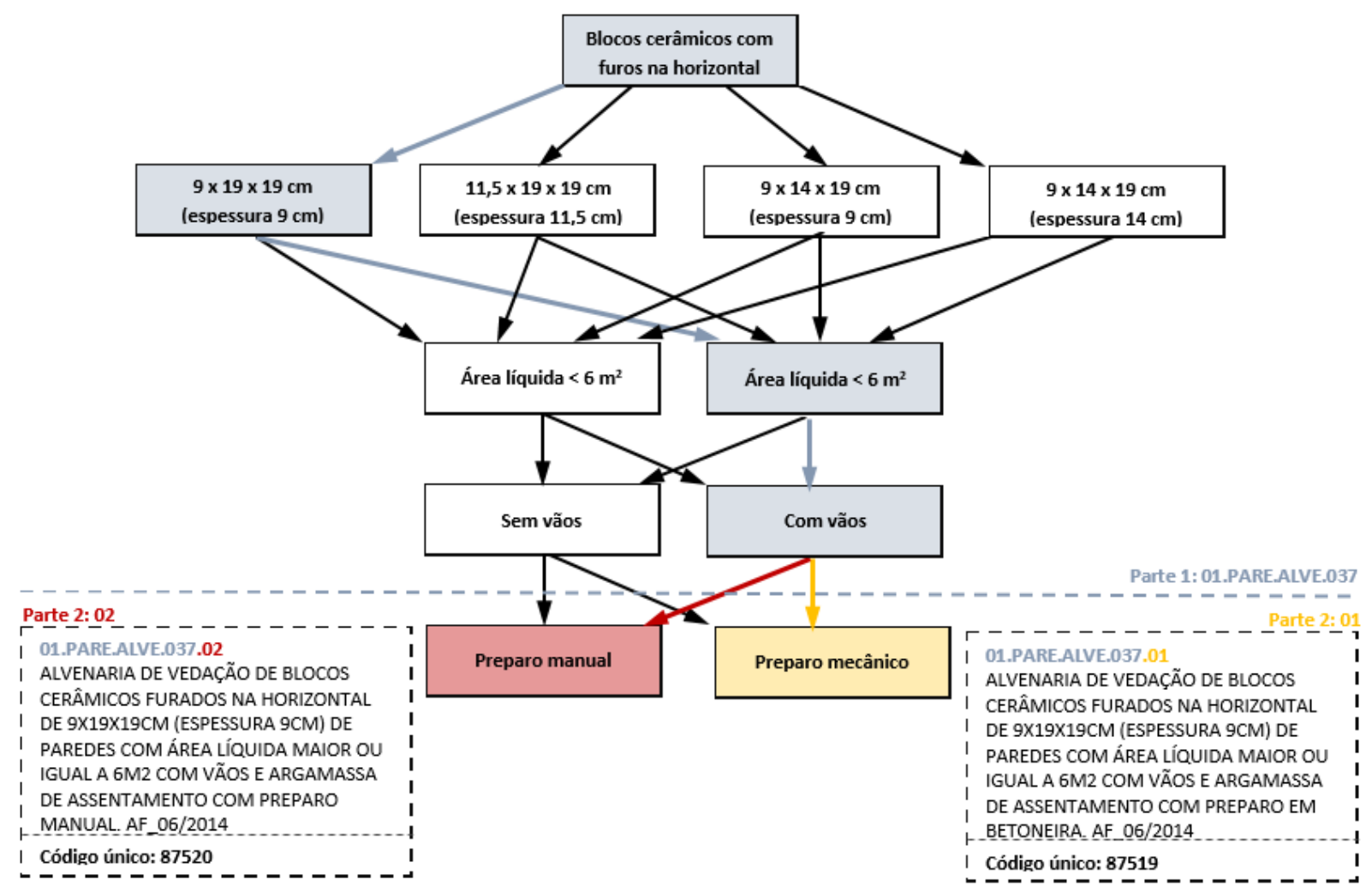

Figura 1: Trechos do Caderno Técnico de Alvenaria de Vedação.

Os materiais relacionados a alvenaria de vedação possuem o código "XX.PARE.ALVE.YYY/ZZ", onde XX, YYY e ZZ são números, "XX.PARE.ALVE.YYY" está associado a primeira parte da árvore (cada escolha de caminho pode gerar valores para $X X$ e YYY diferentes) e ZZ está associado a folha da árvore, fechando o caminho completo e determinando os códigos únicos (completo e numérico). Portanto, a expressão "PARE.ALVE" consegue representar qualquer alvenaria de vedação existente no SINAPI, sem a definição de antemão de suas especificações. O código único é a base do modelo codificado, enquanto a expressão desvinculada de código é a base do modelo com parâmetros de texto, ambos detalhados a seguir.

\subsection{Fluxograma do processo}

O fluxograma apresentado na Figura 2(a) mostra o primeiro método de modelagem aplicado na CAIXA. Nos trabalhos iniciais, atribuía-se o código único de composição ou insumo SINAPI a cada parte do modelo (no campo nota-chave do software utilizado). Essa metodologia, que se mostrava muito dispendiosa de tempo e esforço, além de estar sujeita a erros em função do processo manual no qual se encontrava, apresentava a necessidade de consultar cada parâmetro atribuído ao código SINAPI de forma manual. Por exemplo, para composições de alvenaria era necessário avaliar: espessura da parede, material do bloco, forma de preparo da argamassa, área do vão, existência de portas e janelas. A combinação desses parâmetros gerava um código SINAPI, o qual era utilizado para alimentar o modelo BIM. A equipe o chama de Modelo Codificado. 


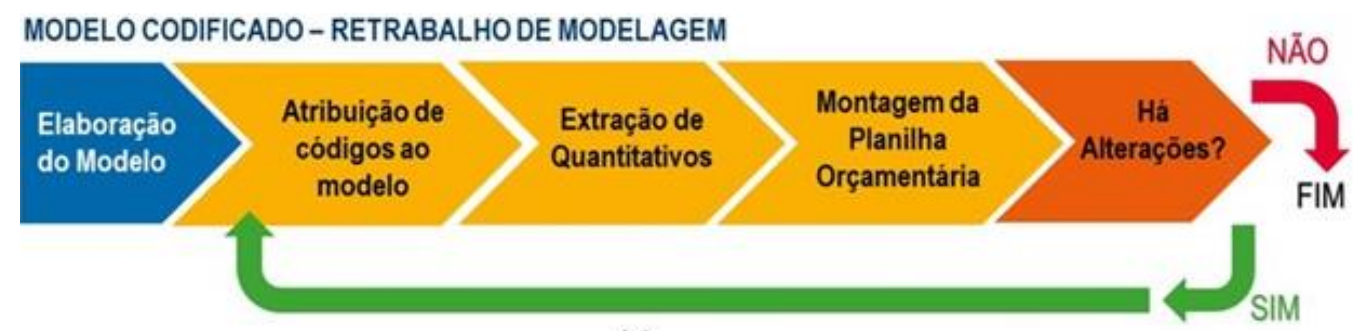

(a)

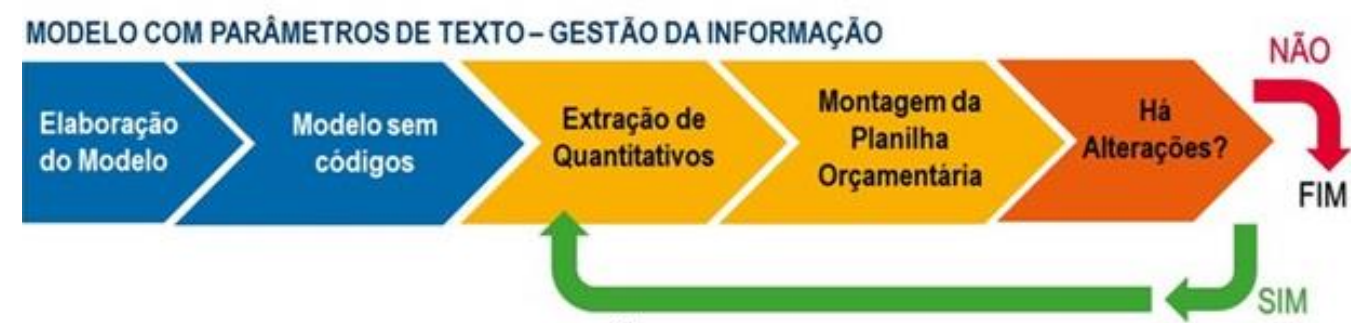

(b)

Figura 2: Fluxograma do processo de modelagem.

Ao longo do tempo de trabalho, a equipe foi descobrindo possibilidades de aprimorar a forma de gerir a informação para além da modelagem e, em 2018, momento em que foi formalizado um grupo de trabalho com objetivo específico de tratar do tema BIM, foi possível avançar bastante após um período de discussões no assunto.

Além de ampliar o registro da metodologia utilizada até então na CAIXA, durante o desenvolvimento dos trabalhos sobre modelagem em BIM, foi também mapeada a possibilidade de utilização da metodologia de parâmetros de texto a partir da dissertação de mestrado de FELISBERTO [8]. Tal trabalho buscou equilibrar o esforço de modelagem e a quantificação 2D por meio de ferramenta gerenciadora de informações (no caso, o autor utilizou as tabelas de software BIM para compatibilização). Observou-se, inclusive, que, ainda que fosse necessária a modelagem, esta poderia ocorrer com poucos elementos e de Nível de Desenvolvimento (LOD) menos elevado.

O referido trabalho foi fundamental para inspirar a organização do novo template desenvolvido em software de modelagem BIM autoral que partiu de uma nomenclatura alternativa à anterior (que utilizava o código SINAPI). Tal nomenclatura possui caráter genérico e é definida por meio de parâmetros de texto, insere-se no modelo e permite a desvinculação do código específico do SINAPI. O fluxograma da Figura 2(b) mostra essa metodologia de modelagem sem códigos. A equipe o chama de Modelo com Parâmetros de Texto.

Importante salientar que, apesar de o Nível de Desenvolvimento (LOD - Level of Development) ser menos elevado, o Nível de Informação (LOI - Level of Information) continua sendo bastante elevado na metodologia proposta.

\subsection{Detalhamento da metodologia com parâmetros de texto}

A partir de 2018, foi elaborada uma proposta de nomenclatura simplificada, que continuou sendo baseada nos Cadernos Técnicos de Composições de Custo do SINAPI [9], porém utilizou apenas parte da informação ali disponibilizada. De forma a explicar a forma de trabalho proposta, a Figura 3 ilustra a biblioteca de materiais, que é utilizada como um repositório para os insumos e composições a serem cadastrados. Utiliza-se o campo "Descrição" para inserir a 
nomenclatura em formato genérico. Nessa metodologia, os parâmetros SINAPI estão mais ligados à biblioteca de materiais e não à criação de componentes da família.

Navegador de materiais - .ALVENARIA DE VEDAÇÃO BLOCO CERÂMICO 9X19X19CM

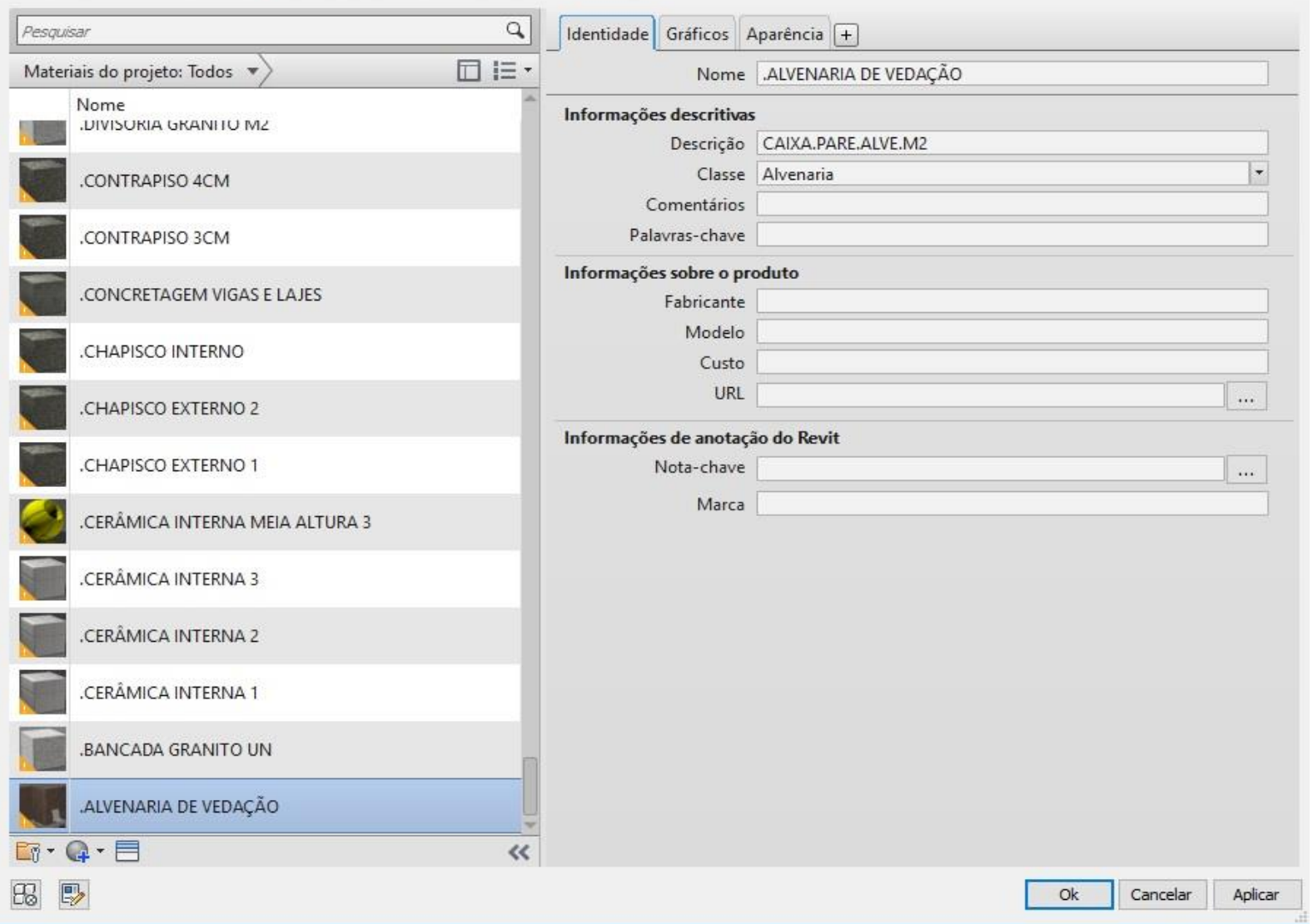

Figura 3: Biblioteca do software autoral com nomenclatura genérica no campo "Descrição".

No campo "Descrição" a nomenclatura de cada componente é composta da estrutura P1.P2.P3, onde P1 refere-se a identificação de origem do elemento, P2 é o prefixo do caderno técnico de composições SINAPI, e P3 é a unidade de medida. A Figura 3 exemplifica a nomenclatura proposta.

A primeira palavra da estrutura da nomenclatura (P1) é CAIXA para o caso específico da base SINAPI, sendo que outras bases de dados também podem ser utilizadas. A equipe está em fase de preparo para compartilhamento do material e este procedimento visa melhorar a rastreabilidade em caso de publicação.

Em continuidade, a segunda parte da nomenclatura do elemento $(\mathrm{P} 2)$ é composta pelo prefixo proposto no próprio Caderno Técnico de Composições de Custo SINAPI, de forma a aproveitar um código de classificação já existente, porém sem o sufixo. Por exemplo, ao invés de identificar o elemento como 01.PARE.ALVE.037/01 (que gera o código SINAPI 87519 único), passa-se a identificá-lo por meio de apenas "PARE.ALVE", o que implica que o item se refere a genericamente a uma alvenaria de vedação. Os parâmetros específicos deste item em projeto possibilitam a atribuição correta ao código único SINAPI. 
¿CAIXA-QUANTITATIVO MATERIAL PAREDE>

\begin{tabular}{|c|c|c|c|c|c|c|}
\hline A & $B$ & C & D & E & $\mathbf{F}$ & G \\
\hline Descriç̧o material & Sigias & Area & Coenentários & Material: SinLAPI Geral & Largura & SINAPI Gera \\
\hline ALVENAQIA DE VEDAC,DO & CANCA.PARE.ALVE.M2 & $0,01355 \mathrm{~m}^{2}$ & COM VÁOS & PREPARO NUTOMÁTICO & 0,090 & BLOCO CERÁWICO \\
\hline . ALVENARIA DE VEDACZOO & CANXA.PARE.ALVE.M2 & $0,18200 \mathrm{~m}^{2}$ & SEn víos & PREPARO NUTOMÁTICO & 0,090 & BLOCO CERÁUICO \\
\hline .ALVENARIA DE VEDACÃOO & CAXX.PAQE.ALVE.MZ & $0,22983 \mathrm{~m}^{2}$ & SEM VÁOS & PREPARO AUTOMÁTICO & 0,090 & BLOCO CERÁWICO \\
\hline ALVENAARIA DE VEDACCAOO & CANXA.PADE.ALVE.MZ & $0,24900 \mathrm{~m}^{2}$ & SEM VÃOS & PREPARO NUTOMÁTICO & 0,090 & BLOCO CERÁMLCO \\
\hline AL VENARIA DE VEOACAOOO & CAXXAPARE.ALVE.M2 & $0,25600 \mathrm{~m}^{2}$ & SEM VALOS & PREPARO AUTOMÁTICO & 0,090 & BLOCO CERAMICO \\
\hline . ALVENARIA DE VEDACAO & CAXA.PARE.ALVE.M2 & $0,31200 \mathrm{~m}^{2}$ & SEM vios & PREPARO AUTOMÁTICO & 0,090 & BLOCO CERÁNACO \\
\hline .ALVENAQIA DE VEDACAO & CANXAPADE.ALVE.MZ & $0,78260 \mathrm{~m}^{2}$ & SEM VÁOS & PREPARO NUTOMÁTICO & 0,090 & BLOCO CERÁMICO \\
\hline ALVENARIA DE VEOACZOD & CANXA,PARE.ALVE,MZ & $1,16246 \mathrm{~m}^{2}$ & SEM VÄOS & PREPARO AUTOMÁTICO & 0,090 & BLOCO CERÁMICO \\
\hline ALVENARIA DE VEOAC,ÁO & CANXA,PARE.ALVE.M2 & $1,29745 \mathrm{~m}^{2}$ & SEM VÁOS & PREPARO NUTOMÁTICO & 0,090 & BLOCO CERÁMICO \\
\hline ALVENARIA DE VEOACAO & CAIXAPADE.ALVE.M2 & $1,96000 \mathrm{~m}^{2}$ & COM VÄOS & PREPARO AUTOMÁTICO & 0,090 & BLOCO CERÁMLO \\
\hline . ALVENARIA DE VEOACAOO & CAXX.PARE.ALVE.MZ & $2,10028 \mathrm{~m}$ & SEM VÃOS & PREPARO AUTOMÁTICO & 0,090 & BLOCO CERÁULCO \\
\hline ALVENARIA DE VEDACCAO & CANXA.PARE.ALVE.MZ & $2,28995 \mathrm{~m}^{2}$ & SEM VÁOS & PREPARO NUTOMÁTICO & 0,090 & BLOCO CERÁMLO \\
\hline . AL VENARIA DE VEOAC,ZO & CANXA.PARE.ALVE.MZ & $2,38041 \mathrm{~m}^{2}$ & SEM VAOS & PREPARO NUTOMÁTICO & 0,090 & BLOCO CERÁNACO \\
\hline . ALVENARIA DE VEDACAOO & CAXA.PARE.ALVE.M2 & $2,41190 \mathrm{~m}^{2}$ & SEM VÃOS & PREPARO NUTOMÁTCCO & 0,090 & BLOCO CERÁMICO \\
\hline ALVENARIA DE VEOACAO & CANXAPARE.ALVE.MZ & $3,00006 \mathrm{~m}^{2}$ & SEU VÁOS & PREPARO NUTOMÁTICO & 0,090 & BLOCO CERÁNLO \\
\hline ALVENARIA DE VEDACIO & CANXA.PARE.ALVE.MZ & $3.01325 \mathrm{~m}^{2}$ & SEM víos & PREPARO NUTOMÁTICO & 0,090 & BLOCO CERÁMICO \\
\hline .ALVENARIA DE VEOACZ̃OO & CANXAPARE.ALVE.MZ & $3,05390 \mathrm{~m}^{2}$ & SEM VÃOS & PREPARO NUTOMÁTICO & 0,090 & BLOCO CERÁUICO \\
\hline .ALVENARIA DE VEOACAOO & CAXXA.PAPE.ALVE.M2 & $4,10963 \mathrm{~m}^{2}$ & SEM VÁOS & PREPARO NUTOMÁTICO & 0,090 & BLOCO CERAMUICO \\
\hline . ALVENARIA DE VEOACAO & CAIXA,PARE.ALVE,M2 & $4,15239 \mathrm{~m}^{2}$ & SEM VÁOS & PREPARO AUTOMÁTICO & 0,090 & BLOCO CERÁMICO \\
\hline . ALVENARIA DE VEDACAO & CAXA.PARE.ALVE.MZ & $4,20055 \mathrm{~m}^{2}$ & SEM VÁOS & PREPARO NUTOMÁTICO & 0,090 & BLOCO CERÁLICO \\
\hline . ALVENARIA DE VEDACAOO & CANXA.PAQE.ALVE.MZ & $4,24122 \mathrm{~m}^{2}$ & SEM víos & PREPARO AUTOMÁTICO & 0,090 & BLOCO CERÁMICO \\
\hline ALVENARIA DE VEDAC,ZO & CANXA.PARE.ALVE.MZ & $6,11893 \mathrm{~m}^{2}$ & SEM VÁOS & PREPARO NUTOMÁTICO & 0,090 & BLOCO CERÁMICO \\
\hline ALVENAQIA DE VEOACAO & CAXXAPARE.ALVE.MZ & $6,18007 \mathrm{~m}^{2}$ & SEM VAOSS & PREPARO AUTOMÁTICO & 0,090 & BLOCO CERÁMLCO \\
\hline . ALVENARIA DE VEDACAO & CAXXA.PADE.ALVE.MZ & $6,20995 \mathrm{~m}^{2}$ & SEM VÁOS & PREPARO AUTOMÁTICO & 0,090 & BLOCO CERÁMICO \\
\hline .ALVENARIA DE VEDACÁO & CANXAPARE.ALVE.M2 & $6,36184 \mathrm{~m}^{2}$ & SEM VÃOS & PREPARO NUTOMÁTICO & 0,090 & BLOCO CERÁUICO \\
\hline . ALVENARIA DE VEOACAOO & CAIXA.PARE.ALVE.MZ & $6,89020 \mathrm{~m}^{2}$ & COM VÁOS & PREPARO NUTOMÁTICO & 0,090 & BLOCO CERAMICO \\
\hline
\end{tabular}

Figura 4: Tabela software de modelagem BIM autoral contendo parâmetros de composições de Alvenaria.

A título de ilustração, considere a tabela mostrada na Figura 4, gerada automaticamente a partir do modelo, que especifica itens de alvenaria de vedação (que se enquadram na expressão "PARE.ALVE" de P2) no projeto, onde a coluna B contém a aplicação da nomenclatura genérica e as colunas $\mathrm{C}, \mathrm{D}, \mathrm{E}, \mathrm{F}$ e G contém os valores dos parâmetros da composição de alvenaria. Usando a combinação dos valores das colunas $\mathrm{C}$ a $\mathrm{G}$, é possível determinar o caminho único a ser percorrido na estrutura de árvore da Figura 1 e, consequentemente, a especificação do código único SINAPI.

Observa-se que é necessário criar parâmetros que não são padrão do software utilizado e, ao mesmo tempo, utiliza-se também outros parâmetros que já são padrão do software.

Finalmente, a terceira parte da nomenclatura (P3) é referente à unidade de medida do insumo ou composição, o que facilita a leitura de informações chave logo no título do elemento.

No Modelo Codificado, todo o conteúdo informacional é detalhado diretamente no modelo. No modelo com Parâmetros de Texto, a nomenclatura genérica permite a escolha do código SINAPI por meio da combinação de parâmetros. Esses parâmetros são, atualmente, analisados fora do software de modelagem BIM autoral (em planilha Excel) utilizando a ferramenta de filtros.

De forma a exemplificar como se obtém o código único do SINAPI com base na Figura 4, temse, por exemplo, o segmento de parede representado pela primeira linha, com a seguinte combinação de características: área menor que $5 \mathrm{~m}^{2}$, com vãos, com argamassa de preparo automático, largura de $9 \mathrm{~cm}$, material de bloco cerâmico. Essa combinação de 5 parâmetros gera o código SINAPI 87511, conforme caderno técnico de composições exemplificado na Figura 1 .

$\mathrm{O}$ presente trabalho encontra-se em desenvolvimento e entende-se que, futuramente, estes mesmos dados possam vir a ser geridos de forma automatizada via macros, plugins ou outra solução tecnológica. Esta é, inclusive, uma sugestão para trabalhos futuros, de forma a dar continuidade ao conteúdo apresentado e desenvolver o nível de maturidade BIM. 


\section{Estudo de caso}

\subsection{Caracterização do modelo}

Este estudo de caso teve como base o projeto intitulado "Módulo de Educação Infantil", de autoria do Fundo Nacional para o Desenvolvimento da Educação (FNDE) [10].

$\mathrm{O}$ referido projeto, pelo fato de estar publicado, apresentar qualidade em seu detalhamento e disponibilizar todas as disciplinas de forma completa para subsidiar a elaboração de um orçamento, foi escolhido para servir como modelo para estudo de caso deste artigo. $\mathrm{O}$ fato de ter área construída de aproximadamente $180 \mathrm{~m}^{2}$ também o classifica como relativamente pequeno para um equipamento urbano, facilitando as experimentações com uma nova metodologia.

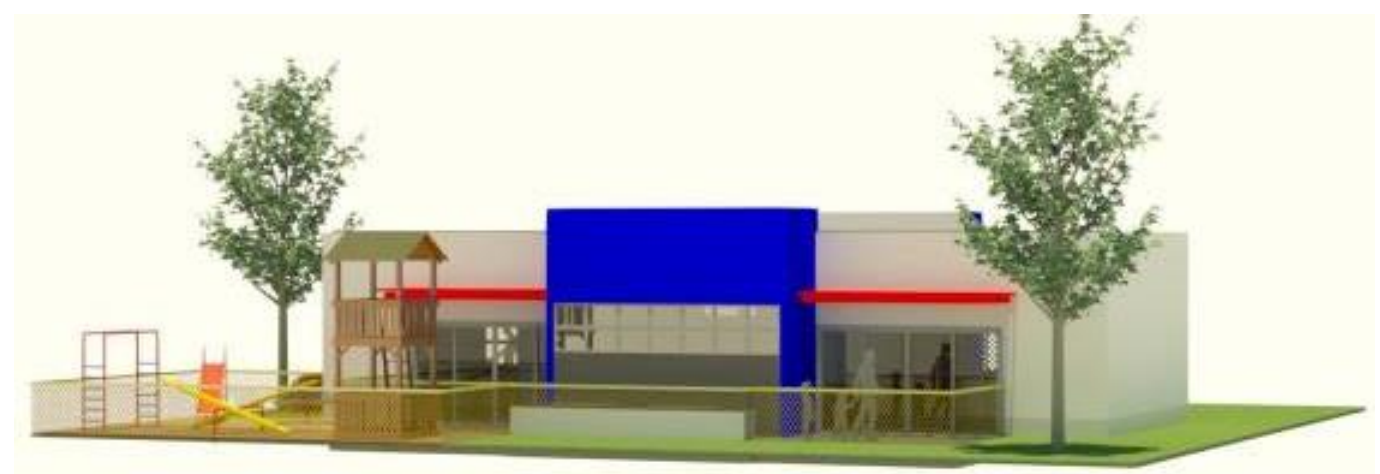

Figura 1: Modelo BIM Módulo Infantil elaborado pela CAIXA baseado em Projeto FNDE.

\subsection{Apresentação de indicadores numéricos}

No contexto deste artigo, para fins de comparação de produtividade, foi realizado um teste cronometrado para a execução de parte de um modelo do Módulo Infantil em software de modelagem BIM autoral de acordo com o método convencional (Modelo Codificado) e outro teste para a execução do mesmo objeto com a mesma ferramenta no método com Parâmetros de Texto (o método proposto e detalhado acima). Desta forma, foram modelados os projetos de arquitetura, estrutura e de fôrmas de uma sala e de parte do banheiro do referido projeto.

O processo de modelagem com a metodologia do Modelo Codificado teve duração de sete horas e vinte minutos, enquanto o processo de modelagem com a metodologia com parâmetros de texto teve duração de quatro horas. A Figura 6 mostra estes tempos de trabalho. O resultado indica que o tempo com o método com parâmetros de texto equivale a $55 \%$ do tempo despendido com o método do modelo codificado.

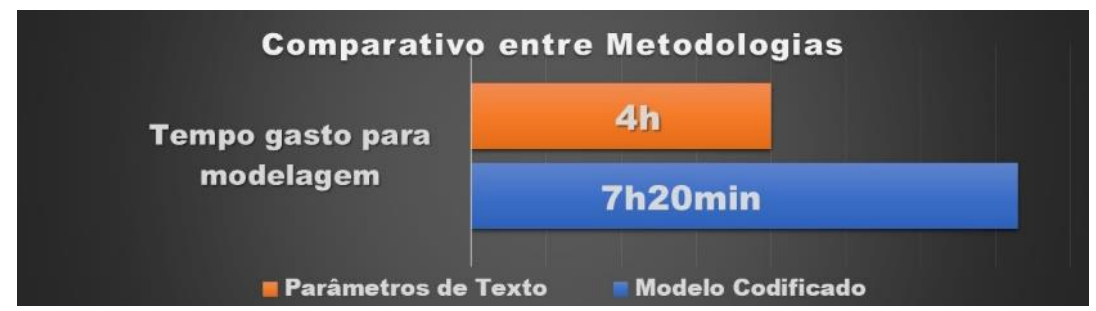

Figura 2 - Comparativo entre Metodologia do Modelo Codificado e Parâmetros de Texto. 


\section{Conclusão}

A forma de nomenclatura proposta neste artigo possibilita uma melhoria no processo de trabalho. A economia de etapas pode ser comprovada visualmente por meio dos fluxogramas apresentados na seção 4.1. De acordo com o estudo de caso, foi gerada uma economia de 55\% no tempo de modelagem. Cabe ressaltar que o ganho de produtividade é relativo ao processo de modelagem exclusivamente, e dentro do contexto de um projeto pequeno de orçamento, não tendo sido contabilizados os tempos de gestão da informação após a conclusão do modelo. Infere-se que ainda continue existindo um aumento na produtividade total, mas que esta seja menor do que a mensurada unicamente na etapa de modelagem.

O SINAPI é um sistema vivo em que ocorre a manutenção regular das composições, que impacta diretamente a Demonstração de Uso das Referências. Usando o método do Modelo Codificado, havia retrabalho de modelagem devido a essas atualizações constantes do modelo BIM. Usando o método com Parâmetros de Texto, elimina-se o retrabalho devido à desvinculação de códigos específicos SINAPI do modelo BIM. Neste contexto, economiza-se em tempo de modelagem em atividades de rotina regulares.

Somado a este cenário, o mercado da construção civil brasileira tem utilizado o BIM essencialmente para a elaboração de modelos voltados para projeto, que não são adequados para orçamentos/estimativas de custos. $\mathrm{O}$ presente trabalho pretende contribuir para $\mathrm{o}$ desenvolvimento do BIM aplicado a Engenharia de Custos considerando o potencial avanço que esta forma de trabalho pode apresentar para a indústria da Engenharia, Arquitetura e Construção.

\section{Agradecimentos}

Arnaldo Gustavo Andrade Lopes, Mauro Fernando Martins de Castro, Ronaldo Rodrigues, Rômulo de Oliveira, Valdir José Cobos, Ana Alice Engel de Oliveira, Alexandre David Felisberto, Carlos Eduardo Gomes Carneiro, Bruna Cardoso de Sousa e equipe do FNDE.

\section{Notas}

As opiniões expressas neste trabalho são exclusivamente dos autores e não refletem, necessariamente, a visão da Caixa Econômica Federal.

\section{Referências}

[1] BRASIL. (2013, Abr. 08). Decreto $\mathrm{n}^{\mathrm{o}}$ 7983/2013. [Online]. http://www.planalto.gov.br/ccivil_03/_Ato2011-2014/2013/Decreto/D7983.html

[2] BRASIL. (2016, Jun. 30). Lei $\mathrm{n}^{\mathrm{o}}$ 13.303/2016. [Online] http://www.planalto.gov.br/ccivil_03/_ato2015-2018/2016/lei/113303.htm

[3] CAIXA ECONÔMICA FEDERAL, GERÊNCIA NACIONAL DE PADRONIZAÇÃO E NORMAS TÉCNICAS DE GOVERNO, "Demonstrações do Uso do SINAPI" 
CAIXA.gov.br.

http://www.caixa.gov.br/poder-publico/apoio-poderpublico/sinapi/orcamentos-referencia/Paginas/default.aspx (acessado em Fev. 04, 2020).

[4] W. S. Catelani and E. Toledo Santos, "Normas brasileiras sobre BIM," Concreto e Construções, Ano 84, pp. 54-59, Out-Dez 2016. ISSN 1809-7197.

[5] MINISTÉRIO DA INDÚSTRIA, COMÉRCIO EXTERIOR E SERVIÇOS, Estratégia BIM BR: Estratégia Nacional de Disseminação do Building Information Modelling BIM”. Ministério da Indústria, Comércio Exterior e Serviços, Brasília: MDIC, 2018.

[6] W. S. Catelani, "BIM = [inovação na construção], " in Seminário Nacional Arquitetura e Urbanimos: Diálogo com o Futuro, Brasília, DF, Out. 2016. [Online]. Disponível em: http://www.caubr.gov.br/wp-content/uploads/2016/11/WILTON-Brasilia-CAU-BR-WS27-10-2016-Resumed-R1.pdf.

[7] CAIXA ECONÔMICA FEDERAL, GERÊNCIA NACIONAL DE PADRONIZAÇÃO E NORMAS TÉCNICAS DE GOVERNO, Metodologia de Elaboração e Manutenção de Orçamentos de Referência. Edificações - Versão 2. Brasília: CAIXA, 2018.

[8] A. D. Felisberto, "Contribuições para elaboração de orçamento de referência de obra pública observando a nova árvore de fatores do SINAPI com BIM 5D - LOD 300," Dissertação de Mestrado, UFSC, Pós-graduação em Engenharia Civil, Florianópolis, Brasil, 2017.

[9] CAIXA ECONÔMICA FEDERAL, GERÊNCIA NACIONAL DE PADRONIZAÇÃO E NORMAS TÉCNICAS DE GOVERNO, "Estrutura dos Cadernos Técnicos de Composições" CAIXA.gov.br http://www.caixa.gov.br/Downloads/sinapi-manual-demetodologias-e-conceitos/CONHECENDO_ESTRUTURA_CT_11_2019.pdf (acessado em Fev. 05, 2020).

[10]FNDE. "Módulo de Educação Infantil" FNDE.gov.br https://www.fnde.gov.br/index.php/programas/proinfancia/eixos-de-atuacao/projetosarquitetonicos-para-construcao/item/9267-m\%C3\%B3dulo-deeduca\%C3\%A7\%C3\%A3o-infantil (acessado em Fev. 04, 2020). 